\title{
Factores asociados a caídas en el servicio ambulatorio de geriatría de un hospital universitario en Bogotá (Colombia)
}

\author{
Factors Associated with Falls in the Outpatient \\ Geriatric Service of a University Hospital in \\ Bogotá, Colombia
}

Fatores associados a quedas no serviço ambulatorial de geriatria de um hospital universitário em Bogotá, Colômbia

Diego Alejandro Arias Delgado, $\mathrm{MD}^{1 *}$

Laura Cepeda Alfonso, MD ${ }^{1}$

David Coca León, $\mathrm{MD}^{1}$

Diego Chavarro-Carvajal, $\mathrm{MD}^{1}$

Rodrigo Heredia Ramírez, MD ${ }^{1}$

Recibido: 19 de mayo de 2020 • Aceptado: 17 de noviembre de 2020

Doi: https://doi.org/10.12804/revistas.urosario.edu.co/revsalud/a.10058

Para citar este artículo: Arias Delgado DA, Cepeda Alfonso L, Coca León D, Chavarro-Carvajal D, Heredia Ramírez R. Factores asociados a caídas en el servicio ambulatorio de geriatría de un hospital universitario en Bogotá (Colombia). Rev Cienc Salud. 2021;19(1):1-11. https://doi.org/10.12804/revistas.urosario.edu.co/revsalud/a.10058

\section{Resumen}

Introducción: las caídas en el adulto mayor influyen de manera negativa en su estado funcional y el riesgo de caídas se eleva progresivamente con la edad, por lo que es importante objetivar todos los factores de riesgo de cada paciente. El objetivo fue describir la prevalencia y los factores asociados con caídas en los

1 Unidad de Geriatría, Hospital Universitario San Ignacio (Colombia); Instituto de Envejecimiento, Pontificia Universidad Javeriana (Colombia). https://orcid.org/0000-0003-0508-8083

Diego Alejandro Arias Delgado: ORCID https://orcid.org/0000-0003-0508-8083

Laura Cepeda Alfonso: oRCID https://orcid.org/0000-0002-1712-2237

David Coca León: orCID https://orcid.org/0000-0001-7088-0400

Diego Chavarro-Carvajal: oRCID https://orcid.org/0000-0002-8584-3191

Rodrigo Heredia Ramírez: oRCID https://orcid.org/0000-0002-7681-0355

Autor de correspondencia: daariasd@javeriana.edu.co 
pacientes del servicio ambulatorio de geriatría del Hospital Universitario San Ignacio en Bogotá (Colombia). Materiales y métodos: se realizó un estudio descriptivo con pacientes valorados en la consulta externa de geriatría del Hospital Universitario San Ignacio desde enero de 2017 hasta diciembre de 2018. Resultados: se incluyeron 233 pacientes, en quienes se documentó una prevalencia de caídas del 44.5\%. En el análisis bivariado, las variables con asociación estadísticamente significativa fueron: la presencia de trastorno neurocognitivo mayor (TNCM) (OR: 2.31; Ic 95\%: 1.28-4.18; p: 0.005), el miedo a caer (oR: 2.27; IC 95\%: 1.24-4.18; p: 0.008), un grado de dependencia moderado a total (oR: 1.93; Ic 95\%: 1.11-3.35; p: 0.019) y un mal estado nutricional (oR: 1.18; ic 95\%: 1.099-3.18; p: 0.02). No obstante, en el análisis multivariado, las variables que mantuvieron la asociación fueron el TNCM (oR: 2.09; ic 95\%: 1.14-3.86; p: 0.001) y el miedo a caer (OR: 2.27; IC $95 \%$ : 1.19-4.32; p: 0.001). Conclusión: las variables asociadas con caídas en nuestra población se relacionan con lo descrito en la literatura mundial; son necesarias intervenciones para corregir estos factores y evitar caídas, así como diseñar más estudios para detectar otros factores asociados.

Palabras clave: ancianos; caídas; pacientes ambulatorios; valoración geriátrica integral; características sociodemográficas.

\section{Abstract}

Introduction: Falls in the elderly have a negative impact on functional status and the risk of falls increases progressively with age, so it is important to objectify all the risk factors of each patient. Our objective was to describe the prevalence and factors associated with falls in patients from the geriatric outpatient service of the San Ignacio University Hospital in Bogotá, Colombia. Materials and methods: A descriptive study was carried out with patients assessed in the geriatric outpatient clinic of the San Ignacio University Hospital from January 2017 to December 2018. Results: 233 patients were included, documenting a prevalence of falls of $44.5 \%$. In the bivariate analysis, the variables with a statistically significant association were: the presence of major neurocognitive disorder (MNCD) (OR: 2.31; $95 \%$ cr: 1.28 4.18; p: 0.005), fear of falling (oR: 2.27; $95 \%$ cr: 1.24-4.18; p: 0.008), a moderate to total degree of dependence (oR: 1.93; $95 \%$ cr 1.11-3.35; p: 0.019) and a poor nutritional status (OR: 1.18; $95 \%$ cr: 1.099-3.18; p: 0.02). However, in the multivariate analysis, the variables that maintained the association were MNCD (OR: 2.09; $95 \%$ cr: 1.14-3.86; p: 0.001) and fear of falling (oR: 2.27; $95 \%$ cr: 1.19-4.32; p: 0.001). Discussion: The variables associated with falls in our population are related to what is described in the world literature; Interventions are necessary to correct these factors and avoid falls in our population, as well as to design more studies to detect other associated factors.

Keywords: Elderly; accidental falls; outpatiens; comprehensive geriatric assessment; sociodemographic characteristics.

\section{Resumo}

Introdução: as quedas em idosos impactam negativamente o estado funcional e o risco de quedas aumentam progressivamente com a idade, por isso é importante objetivar todos os fatores de risco de cada paciente. Nosso objetivo foi descrever a prevalência e os fatores associados às quedas em pacientes do ambulatório de geriatria do Hospital Universitário San Ignacio em Bogotá, Colômbia. Materiais e métodos: foi realizado um estudo descritivo com pacientes avaliados no ambulatório de geriatria do Hospital Universitário San Ignacio no período de janeiro de 2017 a dezembro de 2018. Resultados: foram incluídos 233 pacientes, documentando uma prevalência de quedas de 44,5\%. Na análise bivariada, as variáveis com associação estatisticamente significativa foram: presença de trastorno neurocognitivo maior (TNCM) (oR: 2.31; Ic 95\%: 1,28-4,18; p: 0,005), medo de cair (oR: 2,27; ic 95\%: 1,24-4,18; p: 0,008), grau de dependência moderado a total (oR: 1,93; Ic 95\%: 1,11-3,35; p: 0,019) e estado nutricional ruim (oR: 1,18; Ic 95\%: 1,099-3,18; p: 0,02); porém, na análise multivariada, as variáveis que mantiveram a associação foram тNCM (or: 2,09; ic 95\%: 1,14-3,86; p: 0,001) e medo de cair (or: 2,27; ic 95\%: 1,19-4,32; p: 0,001). 
Conclusão: as variáveis associadas às quedas em nossa população estão relacionadas ao que é descrito na literatura mundial; são necessárias intervenções para corrigir esses fatores e evitar quedas em nossa população, bem como desenhar mais estudos para detectar outros fatores associados.

Palavras-chave: idosos; quedas; pacientes ambulatoriais; avaliação geriátrica abrangente; características sociodemográficas.

\section{Introducción}

L as caídas, han sido definidas por Gómez Montes como "eventos en los cuales hay pérdida involuntaria de la estabilidad postural con desplazamiento del centro de gravedad hacia un nivel inferior, usualmente el piso, sin que se vea comprometida la pérdida de la conciencia o el tono postural" (1). La Organización Mundial de la Salud (oms) las considera como un problema de salud pública, pues son la segunda causa mundial de muerte por lesiones no intencionales, solo por debajo de los accidentes de tránsito. (2).

Adicionalmente, son causa importante de discapacidad, pues se han asociado con un descenso entre el $50 \%$ y el $60 \%$ de los años de vida ajustados por discapacidad y años de vida ajustados por calidad de vida. Anualmente, en el mundo se pierden 17 millones de años de vida ajustados por discapacidad en relación con las caídas; entre el $30 \%$ y el $50 \%$ de las caídas desembocan en una lesión de tejidos blandos y 1 de cada 5 caídas termina en lesiones serias, de las cuales el $30 \%$ tiene posterior dependencia funcional (2).

Por todo lo anterior, definir un modelo de estudio que permita diseñar intervenciones para prevenirlas se ha vuelto materia de investigación en los últimos años. En esta medida, se han descrito múltiples factores de riesgo; algunos relacionados con el individuo (intrínsecos) y otros con el entorno (extrínsecos) (1,3). Entre los primeros destacan algunos como los trastornos neurológicos (demencia), efectos adversos (opioides) o presencia de muchos medicamentos (polifarmacia), alteraciones sensoriales (como disminución de la agudeza visual o auditiva) o síndromes clínicos (como la sarcopenia o la malnutrición). Por su parte, dentro de los factores de riesgo extrínsecos caben mencionar la presencia de escaleras, el uso de calzado inadecuado o tapetes.

La ocurrencia de los fenómenos es el resultado de la interacción de todos estos elementos, muchos de los cuales pueden ser fácilmente detectados mediante la valoración geriátrica integral (1); no obstante, aunque son ampliamente conocidos, pocos son los métodos diseñados para predecir la ocurrencia de estos eventos.

De ahí que sea necesario conocer los factores que más se asocian con caídas en la población mayor para preverlas y evitarlas. Por lo tanto, el objetivo de este trabajo es describir la prevalencia de caídas y los elementos de la valoración geriátrica integral que más se asocian 
con estas en los pacientes del servicio ambulatorio de geriatría del Hospital Universitario San Ignacio de Bogotá (Colombia).

\section{Materiales y métodos}

C trató de un estudio descriptivo de corte transversal, en el que se incluyeron todos los - pacientes valorados por primera vez en la consulta externa de geriatría del Hospital Universitario San Ignacio en la ciudad de Bogotá entre el primero de enero de 2017 y el 31 de diciembre de 2018.

Se obtuvieron los datos de las historias clínicas y se consolidaron en una hoja de cálculo de Microsoft Excel® construida con las variables consideradas. La variable dependiente fue "haber presentado una o más caídas en el último año”; como variables independientes se usaron la edad, el sexo, la escolaridad, la polifarmacia (dicotómica, definida como recibir más de 5 medicamentos en el momento de la consulta), la multimorbilidad (dicotómica, más de 5 enfermedades), el miedo a caer (dicotómica, definida como la presencia de algún grado de miedo a caer), el dolor crónico (dicotómica, definida como la presencia o ausencia de algún dolor), la dificultad para caminar por dolor (dicotómica, definida como la presencia o ausencia de la limitación para caminar), el uso de anteojos para ver de lejos, el riesgo nutricional (dicotómica, definida como buen estado nutricional por un puntaje mayor a 11 en el Mini Nutritional Assesment en su forma de tamizaje vs. la presencia de riesgo de malnutrición o malnutrición), la presencia de prefragilidad o fragilidad (dicotómica, definida como un puntaje de la escala FRAIL mayor de 1), un pobre desempeño funcional (dicotómica, un puntaje de la Short Physical Performance Battery [sppв] menor de 9 puntos), el diagnóstico de trastorno neurocognitivo, la dependencia funcional moderada a total (dicotómica, puntaje menor a 60 en el Índice de Barthel) y la institucionalización en el momento de la consulta.

Una vez tabulada la información, mediante el aplicativo STATA 14.0, se obtuvieron frecuencias y porcentajes para presentarla; adicionalmente, se realizó un análisis bivariado para identificar las variables asociadas con caídas y se expresaron en términos de odds ratio (OR), con intervalos de confianza del $95 \%$ y un valor de $p<0.005$ como medidas de significancia estadística. Las variables con asociación estadísticamente significativa fueron llevadas a un análisis multivariado, ajustando por sexo y edad, y presentadas de la misma forma.

El protocolo de investigación del presente estudio fue presentado y aprobado por el Comité Conjunto de Ética en Investigación del Hospital Universitario San Ignacio y de la Facultad de Medicina de la Pontificia Universidad Javeriana y catalogado en la categoría sin riesgo en los términos de la Resolución 8430 de la ley colombiana, con acta de aprobación 01/2018. 


\section{Resultados}

C obtuvo una población final de 233 pacientes después de excluir los duplicados y aqueOllos con información incompleta en la base de datos recogida entre todos los pacientes valorados por primera vez en la consulta externa entre enero de 2017 y diciembre de 2018. Dentro de las características sociodemográficas, se documentó una edad media de 83.3 años. Las mujeres representaron el $67 \%$ de la muestra. Respecto al comportamiento de las variables independientes, encontramos que el $79 \%$ de los pacientes tenía polifarmacia en el momento de la consulta; el 73\%, multimorbilidad; el 59.2\%, riesgo nutricional, y el 51\%, algún grado de temor a caer. El 78\% tuvo un grado de dependencia de moderada a total para actividades de la vida diaria y el $73 \%$ de los individuos tenía como diagnóstico un trastorno neurocognitivo mayor de cualquier etiología.

Se documentó una prevalencia de caídas del $44 \%$ en la muestra general. Cuando se analizó por grupos de edad, se encontró un aumento progresivo con cada decenio. De esta manera, en el grupo entre 65 y 75 años fue del 33\%; en el grupo entre 76 y 85 años, del $42 \%$, y en el grupo de mayores de 85 años, del $54 \%$. En la tabla 1 se registró la información sociodemográfica diferenciada entre los grupos que presentaron caídas o los que no.

Tabla 1. Características demográficas de los pacientes atendidos en el servicio ambulatorio de geriatría del Hospital Universitario San Ignacio

\begin{tabular}{lccc}
\hline \multicolumn{1}{c}{ Variable } & $\begin{array}{c}\text { Al menos una caída en el } \\
\text { último año (n= 106) } \\
\text { Promedio (DE) o n (\%) }\end{array}$ & $\begin{array}{c}\text { Sin caídas (n = 127) } \\
\text { Promedio (dE) o n } \\
\text { (\%) }\end{array}$ & $\begin{array}{c}\text { Valor } \\
\text { de } \boldsymbol{p}\end{array}$ \\
\hline Edad promedio (DE) & $84.32(6.61)$ & $82.48(7.74)$ & 0.55 \\
Mujeres & $69(65)$ & $87(68.5)$ & 0.58 \\
Polifarmacia & $84(79.2)$ & $95(74.8)$ & 0.42 \\
Multimorbilidad & $80(75.4)$ & $90(70.8)$ & 0.61 \\
Miedo a caer & $86(81.1)$ & $83(65.35)$ & 0.007 \\
Dolor crónico & $68(64.1)$ & $76(57.8)$ & 0.50 \\
Limitación para caminar por dolor & $46(43.4)$ & $48(37.9)$ & 0.3 \\
Uso de anteojos para ver de lejos & $56(52.8)$ & $63(49.6)$ & 0.62 \\
Riesgo nutricional & $71(66.9)$ & $66(51.9)$ & 0.02 \\
sppB $\leq 9$ & $98(92.4)$ & $111(87.4)$ & 0.2 \\
$\begin{array}{l}\text { Dependencia moderada a total para activi- } \\
\text { dades básicas de la vida diaria }\end{array}$ & $37(34.9)$ & $69(54.3)$ & 0.002 \\
Fragilidad & & & 0.9 \\
Trastorno neurocognoscitivo mayor & $45(42.4)$ & $54(42.5)$ & 0.005 \\
\hline
\end{tabular}

$\mathrm{SPPB}=$ Short Physical Performance Battery; DE: desviación estándar. 
En el análisis bivariado, las variables que tuvieron una asociación estadísticamente significativa con la presencia de al menos una caída en el último año fueron: la edad (1.03 [0,99-1,07]; $p<0,005$ ), el diagnóstico de тNCM (or: 2.31; ic $95 \%$ : 1.28-4.18; p: 0.005), el miedo a caer (oR: 2.27; ic $95 \%: 1.24-4.18$; p: 0.008), un grado de dependencia para actividades básicas de la vida diaria moderado a total (oR: 1.93; ic $95 \%$ : 1.11-3.35; p: 0.019) y el riesgo nutricional (OR: 1.18; Ic 95\%: 1.099-3.18; p: 0.02), los datos adicionales se presentan en la tabla 2.

Tabla 2. Análisis bivariado

\begin{tabular}{lcc}
\hline \multirow{2}{*}{ Variable } & \multicolumn{1}{c}{ Análisis bivariado } \\
\cline { 2 - 3 } & $\begin{array}{c}\text { Odds ratio } \\
\text { (Intervalo de confianza 95\%) }\end{array}$ & Valor de $\boldsymbol{p}$ \\
\hline Edad & $1.03(0.99-1.07)$ & 0.005 \\
Riesgo nutricional & $1.8(1.099-3.19)$ & 0.02 \\
Dependencia (Barthel <60) & $1.93(1.11-3.35)$ & 0.019 \\
Miedo a caer & $2.27(1.24-4.18)$ & 0.008 \\
Trastorno neurocognoscitivo mayor & $2.31(1.28-4.18)$ & 0.005 \\
Polifarmacia & $1.3(0.84-2.02)$ & 0.22 \\
Sexo & $0.85(0.49-1.48)$ & 0.5 \\
La presencia de algún dolor crónico & $1.2(0.7-2.04)$ & 0.5 \\
El uso de anteojos para ver de lejos & $1.13(0.67-1.9)$ & 0.62 \\
Fragilidad & $1.15(0.5-2.63)$ & 0.729 \\
SPpB $\leq 9$ & $0.91(0.84-0.99)$ & 0.003 \\
Institucionalización & $0.83(0.64-1.08)$ & 0.16 \\
Multimorbilidad & $1.76(0.70-2.27)$ & 0.79 \\
\hline
\end{tabular}

SPPB: Short Physical Performance Battery; DE: desviación estándar.

Una vez hecho el análisis bivariado, usando las variables con asociación estadísticamente significativa con caídas, se realizó un análisis multivariado (ajustando por edad y sexo) y se obtuvo como resultado una asociación estadísticamente significativa para el miedo a caer (oR: 2.27; ic $95 \%$ : 1.19-4.32; p: 0.001) y el diagnóstico de un TNCM (oR: 2.09; Ic 95\%: 1.14-3.86; p: 0.001). 


\section{Discusión}

T as caídas son uno de los grandes síndromes geriátricos; no solo porque se presentan más _comúnmente en este grupo etario, sino por consecuencias como el deterioro funcional y el aumento de la mortalidad. Las caídas tienen una prevalencia considerable. Según algunos autores, en 2018 en Estados Unidos de América fue del $23 \%$ en mayores de 65 años y hasta el $31 \%$ de las personas manifestó tener o haber tenido en el último año alteraciones de la marcha o la estabilidad (3).

En Latinoamérica, la situación no es diferente; los datos oscilan entre el $21 \%$ y el $34 \%$ (4). Específicamente en nuestro país, según datos de la encuesta SABE Colombia de 2015, hasta el 31.9\% de la población mayor de 65 años presentó una caída en el año inmediatamente anterior, sobre todo en el sexo femenino. Este último ha mostrado mayores tasas de admisión hospitalaria, pero una menor tasa de mortalidad, comparadas con las del sexo masculino $(1,5)$.

En nuestro estudio, la prevalencia de caídas fue mayor que la referencia que conocemos para el país (44.5\% vs. $31 \%$ ); sin embargo, en la encuesta SABE Colombia se trató una población más joven (69 años en promedio) y con una menor carga de morbilidad. En otros términos, en comparación con la literatura mundial referente al tema, el comportamiento de nuestra población fue muy similar al descrito previamente; aquí destacan el aumento progresivo de la prevalencia con la edad y una mayor frecuencia en las mujeres, además de otros elementos que se desarrollarán de manera posterior el texto.

Desde hace algunos años se han descrito muchos factores de riesgo para caídas: aquí se incluyen la debilidad muscular, el historial de caídas previas, el déficit de marcha o equilibrio, el uso de ayudas para caminar, el déficit visual, enfermedades (como la artritis, la depresión o el deterioro cognitivo), entre otros (1).

El presente estudio mostró que dos variables tuvieron una asociación significativa, independientemente de los factores de confusión. El primero de ellos es el temor a caer; es un término que se viene estudiando y sobre el cual todavía no existe un consenso, aunque una de las definiciones más aceptadas es la de Tinetti y Powell, para quienes es la "pérdida de la confianza en sí mismo para evitar caídas durante las actividades esenciales" (6). Se ha documentado que puede ser tan prevalente que hasta el $50 \%$ de los ancianos que nunca se han caído lo presenta, así como el $75 \%$ de los que ya presentaron una caída. Cabe aclarar que los datos epidemiológicos pueden variar ampliamente, dado que en el tiempo se han creado múltiples instrumentos de diagnóstico y evaluación $(7,8)$. Su relación con el riesgo de caídas es clara: se ha descrito que aumenta el riesgo hasta 14 veces y nuestros datos son concordantes con esa tendencia $(9,10)$.

En segunda medida, está el diagnóstico de un trastorno neurocognitivo. Esta muestra poblacional en especial no se comportó diferente a lo esperado; aquellos con TNCM sufrieron 2 veces más caídas que aquellos que no lo tenían (or: 2.09; ic 95\%: 1.14-3.86; p: 0.001), y en la literatura, la relación es ampliamente documentada: se ha descrito que las personas ancianas con 
demencia tienen un riesgo de 2 a 3 veces mayor que los ancianos cognitivamente sanos; empero, no se ha identificado la influencia del tipo de demencia en la gravedad y el riesgo de caídas. Las medidas de ejecución física como la velocidad de la marcha o la variabilidad del tiempo de paso son marcadores de riesgo de caídas en pacientes con TNCM (9).

Como se evidenció, muchas de las variables independientes no lograron una asociación estadísticamente significativa. Aquí se encuentran el riesgo nutricional, el número de medicamentos, el uso de anteojos, el dolor crónico, la fragilidad o los bajos puntajes en las pruebas de desempeño físico. Sin embargo, son elementos con evidencia de peso en la literatura que los acreditan como factores de riesgo para caídas; por ejemplo, la malnutrición se ha asociado con un aumento del $12 \%$ en la frecuencia de estos eventos (10), la polifarmacia incrementa el riesgo de caerse 1.5 veces en pacientes frágiles y 2.5 veces en pacientes robustos (11) y el déficit sensorial visual incrementa 2.5 veces el riesgo de padecerlas $(1,12,13)$.

Un hallazgo importante que no estaba contemplado dentro de los objetivos del presente estudio fue la prevalencia de otros tres síndromes geriátricos, como lo son la polifarmacia, la malnutrición y el TNCM. Respecto al primero, se describió una prevalencia del 79\%; sin embargo, cuando se contrastan los datos con una encuesta poblacional, como la encuesta SABE Bogotá de 2012, la diferencia porcentual es de 52 puntos (79 \% vs. 27\%), aunque el grupo de la encuesta era más joven (más del $70 \%$ de los pacientes encuestados tenía menos de 80 años) y probablemente con menos enfermedades (14).

En cuanto al segundo, se identificó riesgo nutricional en el 59.2\% de los pacientes del Hospital Universitario, y cuando se analizaron estos datos a luz de lo que conocemos para nuestro país, se encontró que en SABE Colombia un poco más de la mitad de los pacientes tuvo una condición similar (5); mientras que en SABE Bogotá solo el 38.83\%; y aunque no podemos definir los factores asociados a este desenlace es un punto de partida para futuras investigaciones.

Por último, encontramos la prevalencia del TNCM, que en la población del servicio fue del $73 \%$; sin embargo, los puntos de comparación son pocos. En 2013 se publicó un estudio en la ciudad de Manizales que describió una prevalencia del $39.4 \%$ en población ambulatoria mayor de 65 años, en un trabajo que involucró a 317 pacientes en una área urbana (15). En nuestra institución, por su parte, hallamos en la población hospitalizada en el servicio de geriatría una prevalencia del $68 \%$ de trastorno neurocognitivo de cualquier etiología (16). De alguna manera, esto podría explicar la alta prevalencia de demencia en nuestro servicio de consulta externa, pues un gran porcentaje de los pacientes asiste a control posterior a una hospitalización.

Como fortalezas de este trabajo se debe mencionar que es de los primeros estudios de esta naturaleza que se han hecho con datos provenientes del servicio de consulta externa de geriatría de nuestra institución y que da luces de los factores que se deben intervenir para disminuir el riesgo de caídas en la población adulta mayor del Hospital Universitario 
San Ignacio e incluso de otros centros. Por otro lado, los datos también sacan a la luz las limitaciones de este estudio en particular, dado que tal vez la ausencia de asociaciones significativas entre factores de riesgo ya reconocidos y el desenlace tiene que ver con el tamaño de la muestra, el diseño del estudio o el ajuste de los factores de confusión; adicionalmente, tomando en cuenta que los datos se recopilaron a partir de las narrativas de los pacientes, los cuidadores o los familiares, no se trabajó con una definición estandarizada de caída. Por último, no se recogieron datos acerca de la percepción que los pacientes y sus acudientes tienen sobre la seguridad en su domicilio, puesto que estos instrumentos no se aplican de rutina en la consulta externa de geriatría en el Hospital Universitario San Ignacio.

Concluimos de nuestro trabajo que el síndrome de caídas en la población atendida en nuestra institución es bastante frecuente, pues casi la mitad de los pacientes presentó este evento. También que para esta población en específico los factores que se asociaron con caídas (el temor a caer y el diagnóstico de trastorno neurocognitivo) tienen una prevalencia no despreciable; que existen otros factores sin asociación significativa desde el punto de vista estadístico, pero que si tomamos en cuenta su prevalencia en la población estudiada, podrían estar cumpliendo un papel determinante en el origen de las caídas de nuestros pacientes.

Por todo lo anterior, las caídas deben de ser preguntadas e investigadas en la atención ambulatoria del adulto mayor. La identificación de factores de riesgo y su potencial modificación evitarían que se desemboque en problemas que modifiquen el estado funcional, restrinjan el espacio vital, disminuyan la calidad de vida y aumenten la mortalidad. En este sentido, en nuestra institución usamos la valoración geriátrica integral como una herramienta de abordaje que, mediante una serie de instrumentos, nos permite evaluar sistemáticamente diferentes esferas del estado de los pacientes con el objetivo de detectar condiciones de riesgo no solo para caídas, sino para otros desenlaces. Aquí están incluidos síndromes como la fragilidad, la sarcopenia, la malnutrición, los trastornos cognitivos, la dependencia funcional o la polifarmacia; además, con la valoración de la esfera social es posible descubrir factores de riesgo relacionados con el entorno de los pacientes (extrínsecos). Con todo lo anterior, se diseñan en estrategias específicas para intervenir aquellos factores que son susceptibles de modificación.

Por otro lado, la valoración de los adultos mayores no es asunto solo del geriatra y los mayores grados de complejidad; es importante que desde la atención primaria se busquen de forma activa los factores de riesgo que puedan modificarse para intervenir tempranamente. Aquí cabría resaltar la evaluación no solo del número de medicamentos, del estado nutricional o las comorbilidades, sino también de los factores de riesgo extrínsecos, que son una parte fundamental de la génesis de las caídas.

Asimismo, desde la academia es necesario plantear trabajos con metodologías diferentes que resuelvan las limitaciones mencionadas y que estén enfocados en establecer relaciones causales para facilitar la predicción de estos eventos y evitar los desenlaces adversos que ya se han ilustrado. 


\section{Contribución de los autores}

iego Alejandro Arias Delgado: análisis de los datos y redacción del documento.

Laura Cepeda Alfonso: redacción del documento.

David Coca León: recolección y análisis de los datos.

Diego Chavarro-Carvajal: análisis de los datos.

Rodrigo Heredia: redacción y revisión del documento.

\section{Conflicto de intereses}

Ninguno declarado.

\section{Referencias}

1. Gómez Montes JF. Caídas en ancianos. Manizales, Colombia: Editorial Universidad de Caldas; 2011.

2. Organización Mundial de la Salud. Caídas [internet]. 2018 ene 16 [citado 2020 abr 19]. Disponible en: https://www.who.int/es/news-room/fact-sheets/detail/falls

3. McMurdo MET. "Guideline for the prevention of falls in older persons": essential reading. Age Ageing. 2002;31(1):13-4. https://doi.org/10.1093/ageing/31.1.13

4. Jia H, Lubetkin EI, DeMichele K, Stark DS, Zack MM, Thompson WW. Prevalence, risk factors, and burden of disease for falls and balance or: walking problems among older adults in the U.S. Prev Med. 2019;126:105737. https://doi.org/10.1016/j.ypmed.2019.05.025

5. Marin PP. Latin-American regional review on Falls in older people [internet]. World Health Organization. 2005;1-10. Disponible en: https:/www.who.int/ageing/projects/ AMRO-Chile.pdf?ua=1

6. González Quiñones JC. Resumen: “Sabe Colombia 2015: Estudio Nacional de Salud, Bienestar y Envejecimiento”. Cart Comunitaria. 2017;25(144):24. https://doi.org/10.26752/ ccomunitaria.v25.n144.152

7. Tinetti ME, Powell L. Fear of falling and low self-efficacy: a cause of dependence in elderly persons. J Gerontol. 1993;48(spec issue):35-8. https://doi.org/10.1093/geronj/48. special_issue.35

8. Jung D. Fear of falling in older adults: comprehensive review. Asian Nurs Res. 2008;2(4):214-22. http://dx.doi.org/10.1016/S1976-1317(09)60003-7

9. Pena SB, Guimarães HCQCP, Lopes JL, Guandalini LS, Taminato M, Barbosa DA, et al. Fear of falling and risk of falling: a systematic review and meta-analysis. Acta Paul Enferm. 2019;32(4):456-63. https://doi.org/10.1590/1982-0194201900062 
10. Drootin M. Summary of the updated American geriatrics society/British geriatrics society clinical practice guideline for prevention of falls in older persons. J Am Geriatr Soc. 2011;59(1):148-57.

11. Allali G, Launay CP, Blumen HM, Callisaya ML, De Cock A-M, Kressig RW, et al. Falls, cognitive impairment, and gait performance: results from the Good Initiative. J Am Med Dir Assoc. 2017;18(4):335-40.

12. Saka B, Kaya O, Ozturk GB, Erten N, Karan MA. Malnutrition in the elderly and its relationship with other geriatric syndromes. Clin Nutr. 2010;29(6):745-8. http://dx.doi. org/10.1016/j.clnu.2010.04.006

13. Bennett A, Gnjidic D, Gillett M, Carroll P, Matthews S, Johnell K, et al. Prevalence and impact of fall-risk-increasing drugs, polypharmacy, and drug-drug interactions in robust versus frail hospitalised falls patients: a prospective cohort study. Drugs Aging. 2014;31(3):225-32. https://doi.org/10.1007/s40266-013-0151-3

14. Eriksson S, Strandberg S, Gustafson Y, Lundin-Olsson L. Circumstances surrounding falls in patients with dementia in a psychogeriatric ward. Arch Gerontol Geriatr. 2009;49(1):80-7. https://doi.org/10.1016/j.archger.2008.05.005

15. Hausdorff JM. Gait dynamics, fractals and falls: finding meaning in the stride-to-stride fluctuations of human walking. Hum Mov Sci. 2007;26(4):555-89. https://doi.org/10.1016/j. humov.2007.05.003

16. Cano-Gutiérrez C, Samper-Ternent R, Cabrera J, Rosselli D. Medication use among older adults in Bogota, Colombia. Rev Peru Med Exp Salud Pública. 2016;33(3):419-24. https:// doi.org/10.17843/rpmesp.2016.333.2292

17. Díaz Cabezas R, Marulanda Mejía F, Martínez Arias MH. Prevalencia de deterioro cognitivo y demencia en mayores de 65 años en una población urbana colombiana. Acta Neurol Colomb. 2013;29(3):141-51.

18. Martínez-Velásquez DA, Chavarro-Carvajal DA, García-Cifuentes E, Venegas-Sanabria LC, Cano-Gutiérrez CA. Caracterización de pacientes con demencia avanzada hospitalizados por el servicio de geriatría en un hospital de alta complejidad. Acta Neurol Colomb. 2019;35(1):15-21. https://doi.org/10.22379/24224022228 